\title{
An image enhancement Method for extracting multi-license plate region
}

\author{
Jong-HoYun ${ }^{1}$, Myung-Ryul Choi ${ }^{2}$, and Sang-Sun Lee ${ }^{1}$ \\ ${ }^{\mathbf{1}}$ Department of Electronics and Computer Engineering, Hanyang University, Seoul, Korea \\ [e-mail: sfw1179.yjh@gmail.com, ssnlee@hanyang.ac.kr] \\ ${ }^{2}$ Division of Electronics Engineering, Hanyang University, Ansan, Korea \\ [e-mail: choimy@hanyang.ac.kr] \\ *Corresponding author: Myung-Ryul Choi, Sang-Sun Lee
}

Received October 27, 2016; revised January 7, 2017; revised Febuary 12, 2017; accepted March 6, 2017; published June 30, 2017

\begin{abstract}
In this paper, we propose an image enhancement algorithm to improve license plate extraction rate in various environments (Day Street, Night Street, Underground parking lot, etc.). The proposed algorithm is composed of image enhancement algorithm and license plate extraction algorithm. The image enhancement method can improve an image quality of the degraded image, which utilizes a histogram information and overall gray level distribution of an image. The proposed algorithm employs an interpolated probability distribution value (PDV) in order to control a sudden change in image brightness. Probability distribution value can be calculated using cumulative distribution function (CDF) and probability density function (PDF) of the captured image, whose values are achieved by brightness distribution of the captured image. Also, by adjusting the image enhancement factor of each part region based on image pixel information, it provides a function that can adjust the gradation of the image in more details. This processed gray image is converted into a binary image, which fuses narrow breaks and long thin gulfs, eliminates small holes, and fills gaps in the contour by using morphology operations. Then license plate region is detected based on aspect ratio and license plate size of the bound box drawn on connected license plate areas. The images have been captured by using a video camera or a personal image recorder installed in front of the cars. The captured images have included several license plates on multilane roads. Simulation has been executed using OpenCV and MATLAB. The results show that the extraction success rate is more improved than the conventional algorithms.
\end{abstract}

Keywords: Vehicle Personal Recorder, Car License Plate Extraction, Contrast Enhancement, Cumulative Distribution Function, Computer Vision Sytems 


\section{Introduction}

$\mathbf{R}_{\mathbf{e}}$ equipped with lots of electroinc technologies (Adaptive Cruise Control, Blind Spot Information System, Various sensor technologies, etc.). Among them, a technique for recognizing and analyzing surrounding objects using a camera is being used widely. Automatic License Plate Recognition (LPR) are one of the most important techniques in vehicle image processing which are used to identify a vehicle. LPR algorithms are generally composed of the following three processing steps: location of the license plate region, segmentation of the license plate characters and recognition of each character [1]. The first two steps incorporate image processing techniques on still images or frame sequences (videos) relies on the true recognition rate and the false recognition rate [1]. In addition, LPR algorithms should operate fast enough to fulfill the ITS requirements. In technical terminology, an LPR real-time operation stands for a fast-enough operation not to miss any single interesting target vehicle that moves through the scene [1].

At present, various image processing algorithms have been used in vehicle personal recorders, which are widely equipped in most cars. Vehicle personal recorder images are very useful to provide some evidence for resolving car accidents or crimes, and their image quality is very crucial for the license plate recognition or face recognition. However, it is not possible to maintain consistent image quality due to environmental effects. Therefore, image enhancement algorithm should be one of the important requirements.

Currently, license plate searching methods have resulted in unsatisfied performance for real-time detection due to complicated backgrounds and illumination variations [2]. One research paper has shown that pre-locating vehicles can greatly improve the performance of license plate location [3].

The algorithms used for vehicle location include silhouette-based [4], feature-based [5], and neural network (NN)-based [6] methods. These algorithms have their own characteristics, which provide good results in their respective environments. It is possible to obtain good results when extracting the shape of a vehicle. However, these algorithms cannot provide good results especially when the background is complex and the illumination is weak. Illumination is also one of the manifest factors that affect to extract license plate region [2]. This problem can be resolved by using contrast enhancement method.

Many researchers have developed contrast enhancement algorithms [7]-[14]. Most contrast enhancement algorithms are usually focused and based on the histogram, which provides appropriate contrast information. But, most contrast enhancement algorithms still have some problems, especially when the image brightness is changed excessively [7].

In this paper, we propose a contrast enhancement algorithm for increasing extraction success rate of the license plate region. The general concept of the histogram and conventional algorithms are discussed. And then the proposed algorithm and its simulation results are explained.

\section{Conventional algorithm}

The histogram is the most fundamental tool for many space domain processing techniques. Histogram represents the distribution of brightness and also has the information for contrast. Histogram has been usually used to improve a picture quality. And the intrinsic information is used in image compression and segmentation. Histogram is the scope of the darkest 
domain to the brightest domain of image. In dark image, the components of histogram converge upon low part of brightness scale. And in bright image, the components converge upon its high part. An image with low contrast has the histogram located in the middle of average brightness scale, and is not clear but blurry in black-and-white image. An image with high contrast is distributed over the wide scope of brightness scale, that is, this has a great variety of greyscale value evenly [7]-[9].

Contrast stretching technique is a method that expands the histogram as wide as possible. This is well-applied when histogram distribution of image is Gaussian or similar to Gaussian. The most famous contrast stretching technique is basic light contrast stretching, so called END-IN-SEARCH stretching. This is very effective for the images, whose histogram is concentrated on specific regions. Basic contrast technique can be showed as an equation (1) [7]-[9].

$$
\text { New_Pixel }=\frac{\text { Value }- \text { Low }}{\text { High }- \text { Low }} \times 255
$$

where Value is the current pixel value, Low is the lowest pixel value, and High is the highest pixel value. The subtraction implies that the current value is moved to the lowest pixel value of image, i.e., it plays a role to move its histogram to the left. Basic contrast stretching technique has less effective to improve a picture quality when there is an impulse noise in domain. END-IN-SEARCH stretching retrieves low and high values by deciding a proper thresholds value, i.e., High and Low values can be obtained by eliminating impulse noise value from the histogram of the input image.

DRSHE(Dynamic Range Separate Histogram Equalization) divides the histogram dynamic range into regions at regular intervals and adjusts the size of the grayscale range according to the histogram ratio of the divided areas. The intensity of the histogram is evenly redistributed over the resized grayscale range. DRSHE uses Weighted Average of Absolute color Difference (WAAD) in order to emphasize the edge of the original image and moderate the histogram variation effectively [14].

\subsection{Histogram Equalization [7][11]}

Let $X=\{X(i, j)\}$ denote a given image composed of $L$ discrete gray levels denoted as $\left\{X_{0}\right.$, $\left.X_{1}, \ldots, X_{L-1}\right\}$, where $X(i, j)$ represents an intensity of the image at the spatial location $(i, j)$ and $X(i, j) \in\left\{X_{0}, X_{1}, \ldots, X_{L-1}\right\}$. For a given image $X$, the probability density function $p\left(X_{k}\right)$ is defined as

$$
p\left(X_{k}\right)=\frac{n^{k}}{n}
$$

for $k=0,1, \ldots, L-1$, where $n^{k}$ represents the number of times that the level $X^{k}$ appears in the input image $X$ and $n$ is the total number of samples in the input image. Note that $p\left(X^{k}\right)$ is associated with the histogram of the input image, which represents the number of pixels at a specific intensity $X^{k}$. In fact, a plot of $n^{k}$ vs. $X^{k}$ is known as the histogram of $X$. the cumulative distribution function is defined as 


$$
C D F\left(X_{k}\right)=\sum_{j=0}^{k} p\left(X_{j}\right)
$$

where $X^{k}=x$, for $k=0, l, \ldots, L-1$. Note that $C D F\left(X_{L-1}\right)=1$ by definition[7].

Histogram equalization is a scheme that maps the input image into the entire dynamic range, $\left(X_{0}, X_{\mathrm{L}-1}\right)$ by using the cumulative distribution function as a transform function. That is, let us define a transform function $f(x)$ based on the cumulative distribution function as

$$
f(x)=X_{k} \times \operatorname{CDF}\left(x_{k}\right)
$$

Then the output image of the histogram equalization, $Y$, can be expressed as (5), which is defined as a transfer function, $f\left(X_{k}\right)$. The transfer function, $f\left(X_{k}\right)$, is given by the linear equation of CDF [12].

$$
f\left(X_{k}\right)=\{f(X(i, j) \mid \forall X(i, j))\}=X_{k} \times C D F\left(X_{k}\right)
$$

Fig. 1 shows an original image and the resulted image of the histogram equalization in 256 gray levels. Here we can find that the histogram equalized image is much brighter than the input image
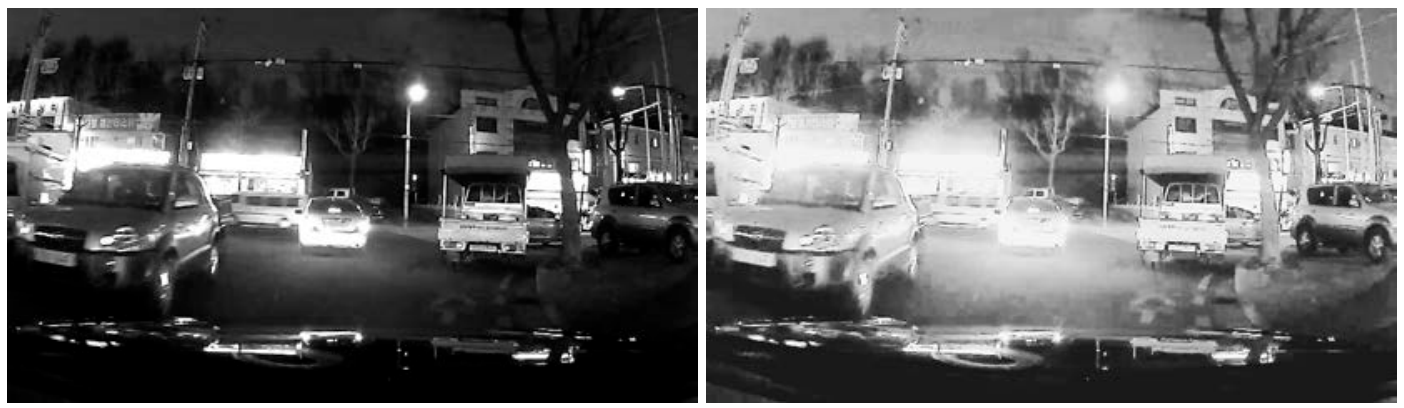

Fig. 1. Simulation results of histogram equalization method for Night street image

\subsection{Bi-Histogram Equalization [11]}

Assume that $X_{m} \in\left\{X_{0}, X_{1}, \ldots, X_{L-1}\right\}$, which is the mean of the image $X$. Based on the mean $X_{m}$, the input image is divided into two subimages $X_{L}$ and $X_{U}$ as

$$
\begin{aligned}
& X=X_{L} \cup X_{U} \\
& X_{L}=\left\{X(i, j) \mid X(i, j) \leq X_{m}, \forall X(i, j) \in X\right\} \\
& X_{U}=\left\{X(i, j) \mid X(i, j)>X_{m}, \forall X(i, j) \in X\right\}
\end{aligned}
$$

Note that the subimage $X_{L}$ is composed of $\left\{X_{0}, X_{1}, \ldots, X_{m}\right\}$ and the other subimage $X_{u}$ is composed of $\left\{X_{m+1}, X_{m+2}, \ldots, X_{L-1}\right\}$. Next, define the respective probability density functions of the subimages $X_{L}$ and $X_{u}$ as 


$$
\begin{array}{cc}
p_{L}\left(X_{k}\right)=\frac{n_{L}^{K}}{n_{L}} & \text { for } k=0,1, \ldots, m \\
p_{U}\left(X_{k}\right)=\frac{n_{U}^{K}}{n_{U}} & \text { for } k=m+1, m+2, \ldots, L-1
\end{array}
$$

$n_{L}^{k}$ and $n_{U}^{k}$ represent the respective numbers of $X_{k}$ in $\{X\}_{L}$, and $\{X\}_{U}$, and $n_{L}$ and $n_{U}$ are the total numbers of samples in $\{X\}_{L}$ and $\{X\}_{U}$, respectively. The respective cumulative density functions for $\{X\}_{L}$ and $\{X\}_{U}$ are then defined as

$$
\begin{aligned}
& C_{L}(x)=\sum_{j=0}^{k} p_{L}\left(X_{j}\right) \\
& C_{U}(x)=\sum_{j=m+1}^{k} p_{U}\left(X_{j}\right)
\end{aligned}
$$

Similar to the case of histogram equalization where a cumulative density function is used as a transform function, let us define the following transform functions exploiting the cumulative density functions

$$
\begin{aligned}
& f_{L}(x)=X_{0}+\left(X_{M}-X_{0}\right) c_{L}(x) \\
& f_{U}(x)=X_{m+1}+\left(X_{L-1}-X_{m+1}\right) c_{U}(x)
\end{aligned}
$$

That is, the output image of the $Y$, is finally expressed as

$$
Y=\{Y\{i, j)\}=f_{L}\left(X_{L}\right) \cup f_{U}\left(X_{U}\right)
$$

Fig. 2 shows an original Night streets image and the processed image of the Bi-Histogram Equalization (BBHE). The simulation results of BBHE show that it cannot solve the washedout appearance problem in overall image due to the excessive change in brightness. Therefore, we have proposed the algorithm to preserve the details of image and also suppresses the over enhancement effectively.
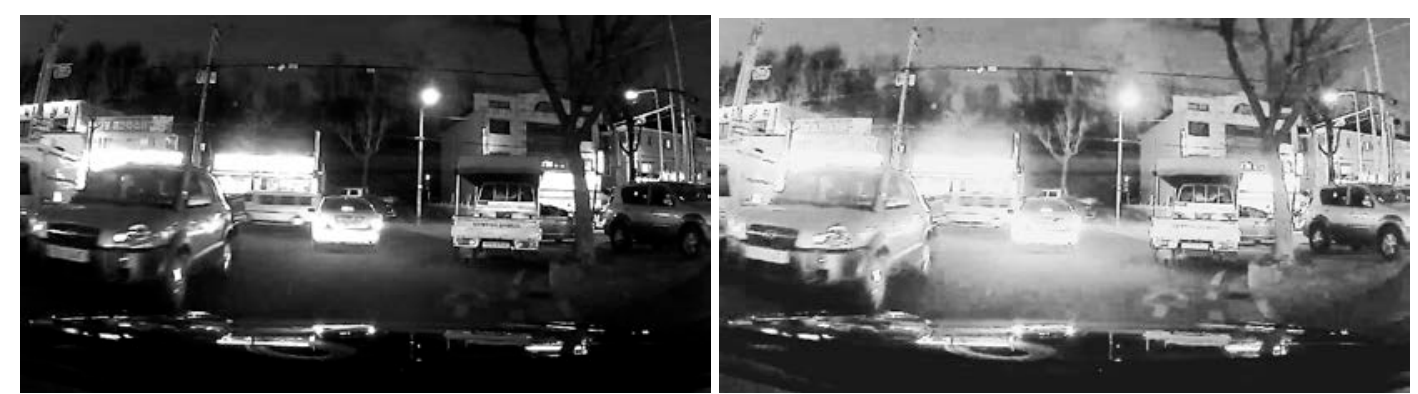

Fig. 2. Simulation results of Bi-histogram equalization method for Night street image

\subsection{Equal Area Dualistic sub-image Histogram Equalization}

An essential element of histogram equalization is to reduce the number of gray levels to 
improve the contrast of the image. In the equalization process, adjacent gray levels with a low probability density are combined, while gaps are occurred between adjacent gray levels with a high probability density. Thus, the image may have uniform gray distribution characteristics. Gray levels with a high probability density increase the dynamic range by equalizing, which enhances image contrast. However, even stretching of the dynamic range can degrade image quality due to rapid changes in image brightness. dynamic range change. This is why the histogram equalization technique is seldom utilized in a video system in the past [12][28].

An image enhancing method, equal area dualistic sub-image histogram equalization (EAD-HE) technique, is put forward in this method [12][24]-[26]. It is expected to eliminate the above drawback effectively. First, the original image is divided into two equal area subimages based on its gray level probability distribution function. Then, the two sub-images are equalized respectively [28]. The output image, Y, is expressed as

$$
Y(i, j)= \begin{cases}X_{0}+\left(X_{e}-X_{0}\right) c_{L}(x) & \text { if } X<X_{e} \\ X_{e}+\left(X_{L+1}-X_{e}\right) c_{U}(x) & \text { else }\end{cases}
$$

where the corresponding cumulative distribution function will be

$$
\begin{array}{ll}
c_{L}(x)=\frac{1}{p} \sum_{i=0}^{k} p_{i} & k=0,1, \ldots \ldots ., e-1 \\
c_{U}(x)=\frac{1}{1-p} \sum_{i=e}^{L-1} p_{i} & k=e, e+1, \ldots \ldots, L-1
\end{array}
$$

In fact, the algorithm can not only enhance the image visual information effectively but also constrain the original image's average luminance from a great shift. But, it is difficult for the EAD-HE technique to implement in real-time processing due to large amounts of computation and storage capacities.

\section{Proposed algorithm}

Proposed algorithm aims to improve the quality of an image so as to search car license plate region. More than 500 moving pictures ( 1 minute, 29 fps/s, 1280x720) have been used from vehicle personal recorders equipped in the car. In addition, it uses a digital camera image taken from the same position as the vehicle personal recorder.

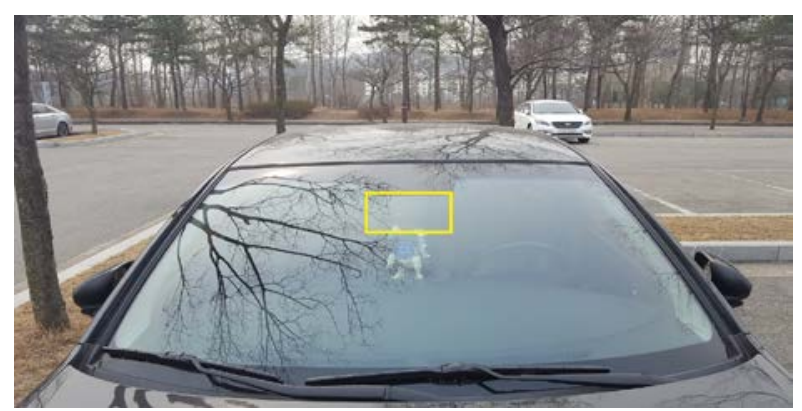

Fig. 3. Location of vehicle personal recorder 

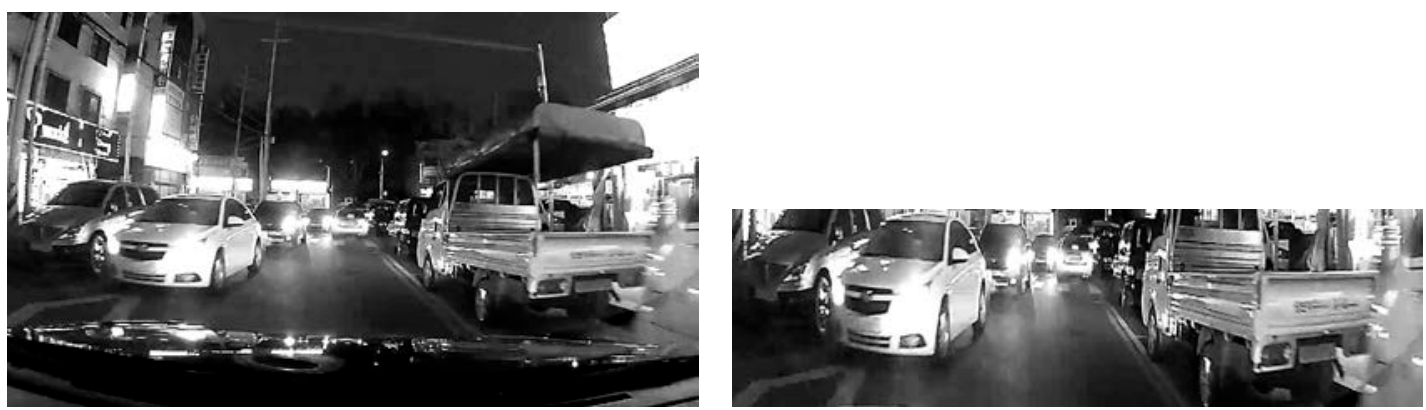

Fig. 4. Original Images and trimmed images

The location of vehicle personal recorder can be seen in the Fig. 3 . The image resolution of vehicle personal recorder is $1280 \times 720$. In order to find the correct license plate region, the unnecessary area was removed based upon the center of the image. Fig. 4 shows the extracted image with resolution 1280x240 from the original image with resolution 1280x720.

Fig. 5 shows the simplified block diagram of the entire proposed algorithm. Input image quality is improved by using the gray distribution of the image. The output gray images are subjected to license plate area extraction algorithm. And the final resulted image contains the blocks with the corresponding captured license plate.

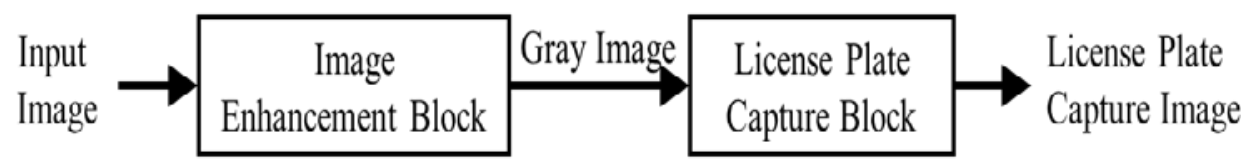

Fig. 5. Block Diagram of Proposed Algorithm

\subsection{Image Enhancement Algorithm.}

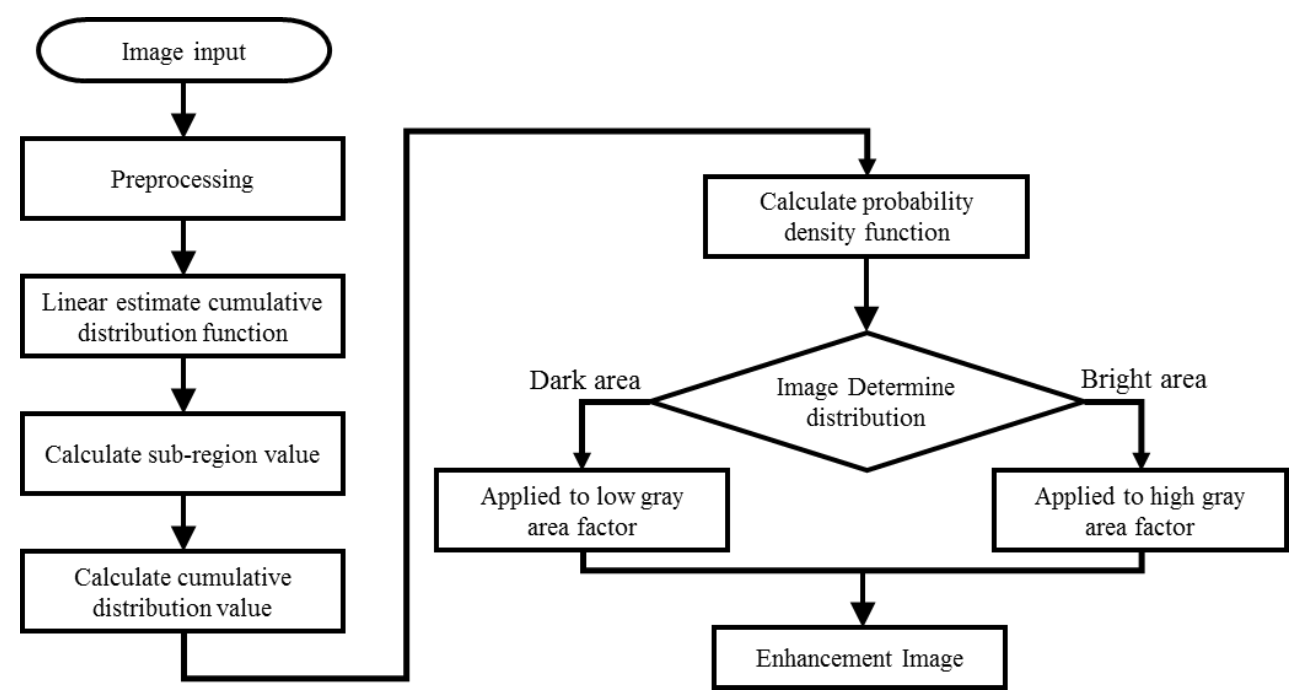

Fig. 6. Flowchart of the proposed Image enhancement algorithm 
In this section, an image enhancement algorithm that can improve an image quality is described, which employs a probability distribution value (PDV) in order to control a sudden change in image brightness. PDV can be obtained by using the cumulative distribution function (CDF) value of the subgroup region. CDF values are calculated by using the first order linear interpolation method for the low computation. For effective processing of the proposed algorithm, the subgroup region value of the CDF has been utilized. In addition, an enhancement method including EF(Enhancement factor) has been proposed. Fig. 6 shows the flow diagram of the image enhancement algorithm process.

The transfer function of Eq. (12) requires computational and hardware complexity due to CDF calculation. To obtain histogram information from an image of $L$ gray levels, it is needed to count the number of pixels for every gray level. The proposed algorithm does not obtain CDF values at all gray levels. Sampling is performed at regular intervals to obtain a $\mathrm{CDF}$ value, and the image brightness level is determined using this value. The function of Eq. (5), $C D F\left(X_{k}\right)$ is the accumulated value up to $K$. On the basis of this, it is possible to calculate linear estimate cumulative distribution function. $C D F\left(X_{k}\right)$ value is used in a linear estimate $\mathrm{m}$ ethod. The proposed CDF is derived from sub-region value (SRV) and its cumulative distribution value (CDV). $S R V_{k}$ is defined as Eq. (14).

$$
S R V_{k}=\frac{L}{S R C} k
$$

for $k=0,1, \cdots, S R C-1$. In the case of 8-bit image, $L=256$, and $S R C$ is total number of subregions, which is chosen to 4 by experimantal results. If the number of sub-regions is bigger than 4 , there was no difference in the improvement of image quality when the number of sub-regions was 4. Also, when the number of sub-regions is less than 4, there is no difference in image quality improvement from the existing algorithm. Then, the $S R V_{k}$ can be used to calculate CDV and $C D V\left(X_{S R V_{k}}\right)$ is the total sum of input image pixels in the range of $X_{S R V_{k}}-\frac{L}{4}$ and $X_{S R V_{k}}$ as follow:

$$
C D V\left(X_{S R V_{k}}\right)=\sum_{j=X_{S R V_{k}}-L / 4}^{S R V_{k}} X_{j}
$$

for $S R V_{k}=\{L / 4, L / 2,3 L / 4, L-1\}$. In this case of 8-bit image, $L=256$ and Eq. (16) is satisfied.

$$
0 \leq C D V\left(X_{\frac{L}{4}}\right) \leq C D V\left(X_{\frac{L}{2}}\right) \leq C D V\left(X_{\frac{3 L}{4}}\right) \leq C D V\left(X_{255}\right)
$$

The CDV values at the subgroup region determine the transfer function, $Y(n)$, by using the first order linear interpolation. Thus, the proposed method can be implemented with much less number of operations. $Y$ is the improved contrast value of an input image $X$, and Eq. (17) means a linear estimate method for the proposed stretching histogram methods.

$$
Y(n)=\left(\alpha X_{S R V_{k}}-\alpha x(n)-1\right) \times C D V\left(X_{S R V_{k}}\right)+\alpha\left(x(n)-X_{S R V_{k}}\right) \times C D V\left(X_{S R V_{k+1}}\right)
$$


for $n=0,1, \ldots, L-1, x(n)$ is the $n$-th input value, where $\alpha=\frac{L}{X_{S R V_{k+1}}-X_{S R V_{k}}}$.

When an input image is in the dark region, the pixels of bright region are excessively brightened by the conventional histogram equalization methods. Thus, when the whole pixels of the input image are either in dark or bright region, the histogram equalization method is applied to input image. However, since a general image has both bright and dark region, the contrast enhancement processing should be done by a different method by distinguishing the bright region from the dark one. The conventional methods have changed the brightness of an input image, which is mainly due to the flattening property of the histogram equalization. It can be also observed that the overall contrast of an input image is degraded after the histogram equalization. A probability distribution function (PDF) is utilized to control a sudden change in image brightness. The PDF provides information for an image characteristic such as contrast and overall intensity distribution of an image.

Proposed algorithm employs PDF to control the excessive brightness changes in the images. It includes a method to control separation of the brightness distribution of the input image. The PDF can be also derived from previously calculated a CDV at subgroup regions and implemented by using simplified operations. The CDF and the PDF can be represented as follows

$$
\begin{aligned}
& C D F(x)=\sum_{i}^{L} P_{i} u\left(x-x_{i}\right) \\
& \operatorname{PDF}(x)=\frac{d}{d x} C D F(x)=\sum_{i}^{L} P_{i} \delta\left(x-x_{i}\right)
\end{aligned}
$$

$P_{i}$ is the probability and $x$ is a random variable. PDF is derived from Eq. (18) using the relationship between the probability density function and the cumulative distribution function. Therefore, the probability distribution value (PDV) can be calculated by using the CDVs of the subgroup regions. Eq. (18) can changed as follow

$$
\operatorname{PDV}\left(S R V_{(k+1, k)}\right)=C D V\left(S R V_{(k+1)}\right)-C D V\left(S R V_{k}\right)
$$

for gray value $k=0, L / 4, L / 2,3 L / 4, L$ and $P D V\left(S R V_{(k+1, k)}\right)$ is a probability density function value of the interval between $k$ and $k+1$. For example, if the value of $\mathrm{k}$ is greater than $L / 2$, the image bright. If the histogram is expanded the two areas in the same method, bright areas will become excessively bright. Then, proposed algorithm is applied to image enhancement factor in order to control the excessive brightness of the bright areas.

The modified transfer function is applied its only dark regions without affecting bright regions. Image enhancement factor can be expressed in Eq. (20) for improving only dark region.

$$
I_{e}=\beta\left|C D V\left(X_{S R V_{k}}\right)-\frac{L}{S R C}\right|
$$

for $\beta=\left(L / S R V_{k}\right)$. 
By Eq. (21), the coefficient of contrast enhancement, EF(Enhancement factor), is defined as follows.

$$
E F\left(X_{S R V}\right)= \begin{cases}C D V\left(X_{S R V}\right) & \text { for no enhancing } \\ I_{e} & \text { for enhancing }\end{cases}
$$

Then, the output function $Y_{n}$ can be expressed in (22) by substituting $C D V$ for $E F$ in Eq. (16).

$$
Y(n)=\left(\alpha X_{S R V_{k}}-\alpha x(n)-1\right) \times E F\left(X_{S R V_{k}}\right)+\alpha\left(x(n)-X_{S R V_{k}}\right) \times E F\left(X_{S R V_{k+1}}\right)
$$

Fig. 6 and 7 show the simulation results of the proposed algorithm and the conventional algorithms such as Histogram Equalization (HI(Fig. 7(b), Fig. 8(b)), Bi Histogram Equalization (BBHE (Fig. 7(c), Fig. 8(c)), Dualistic Sub-Image Histogram Equalization (DSIHE(Fig. 7(d), Fig. 8(d)) and Dynamic Range Separate Histogram Equalization (DRSHE(Fig. 7(e), Fig. 8(e)). Those results show that they do not prevent the washed-out appearance in overall image due to the excessive change in brightness. However, the proposed algorithm (Fig. 7(f), Fig. 8(f)) preserves the details of image and also suppresses the over enhancement effectively.

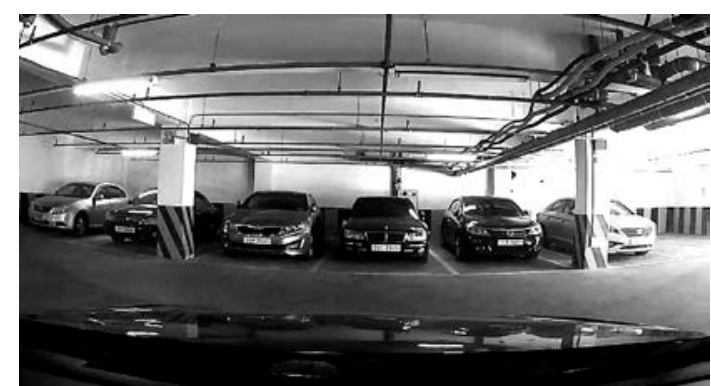

(a) Original Image

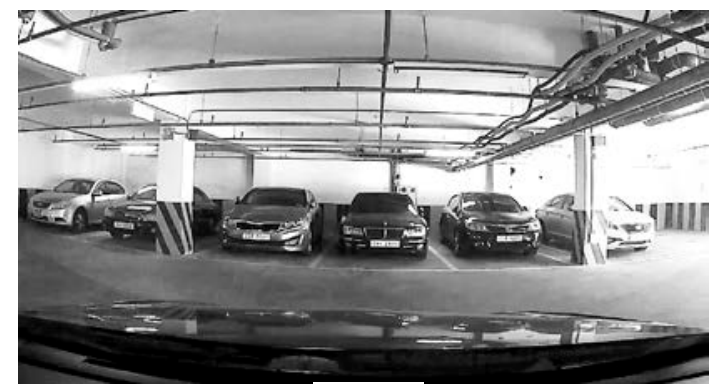

(c) BBHE method

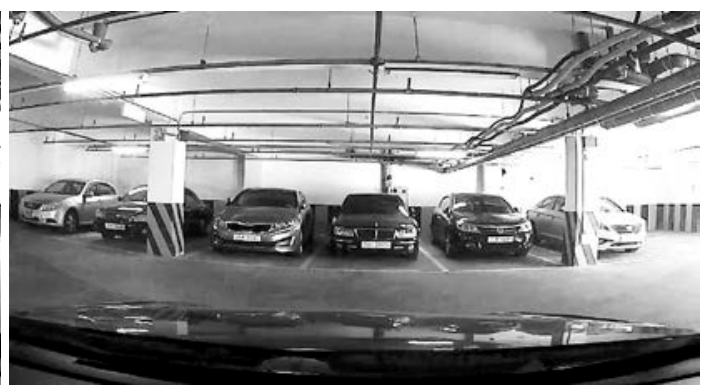

(b) Histogram Equalization method

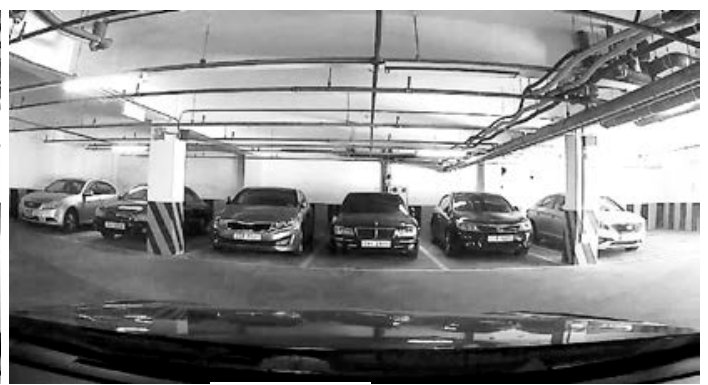

(d) DSIHE method 


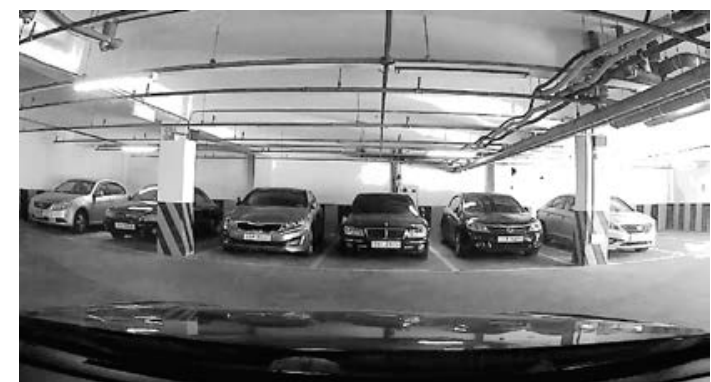

(e) DRSHE method

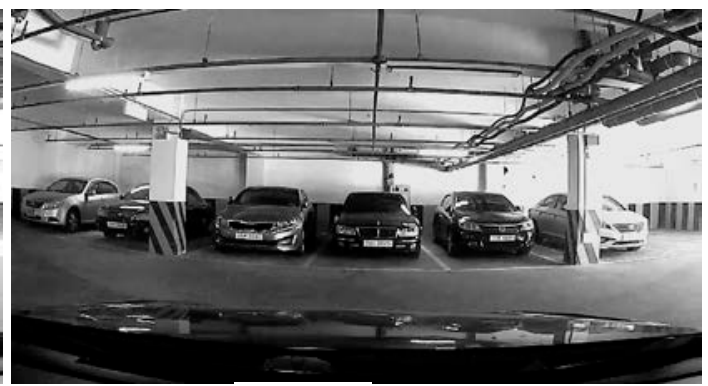

(f) Proposed algorithm

Fig. 7. Simulation results of conventional and proposed method for Underground parking lot

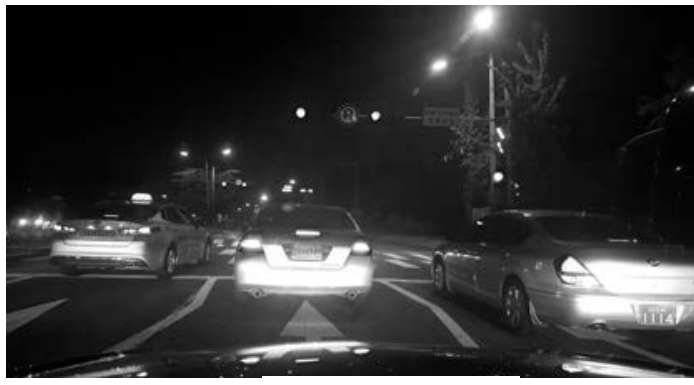

(a) Original Image

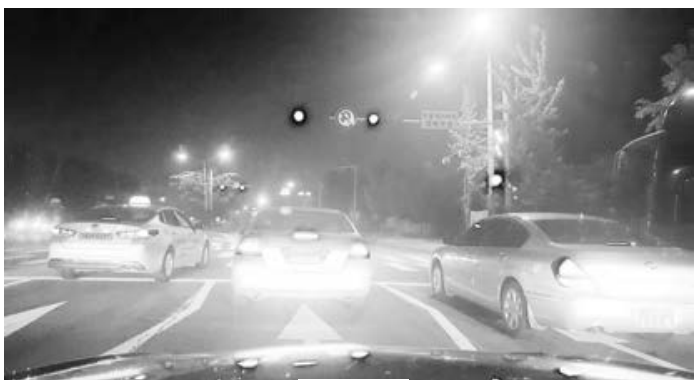

(c) BBHE method

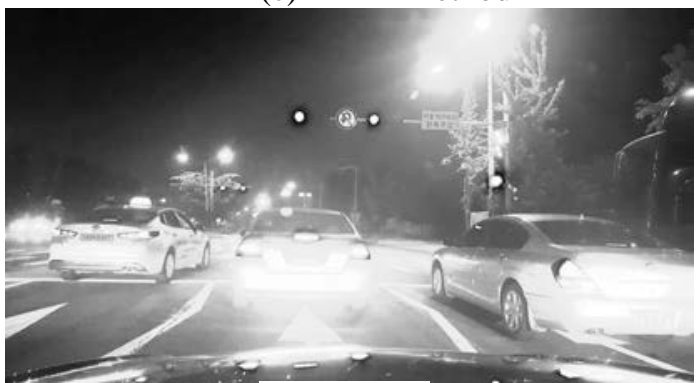

(e) DRSHE method

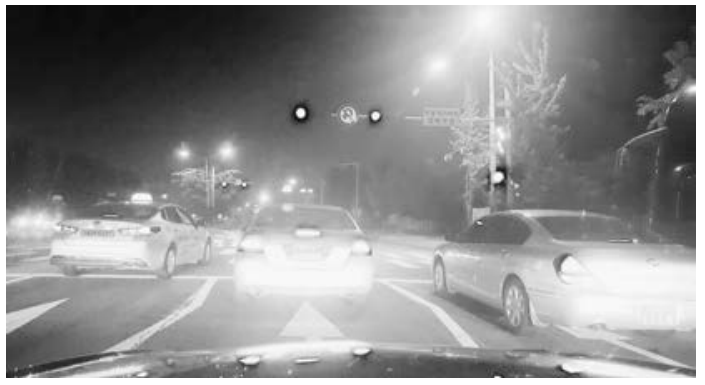

(b) Histogram Equalization method

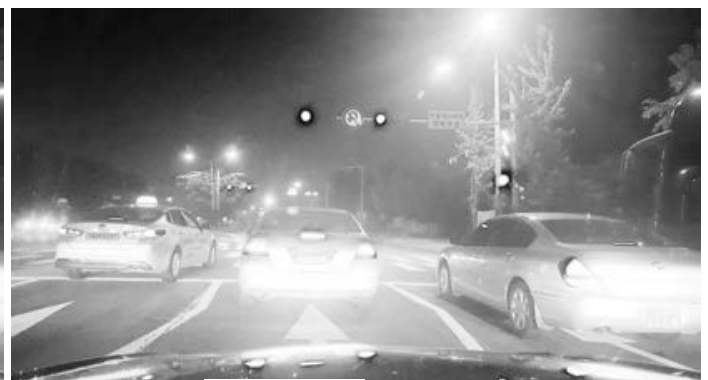

(d) DSIHE method

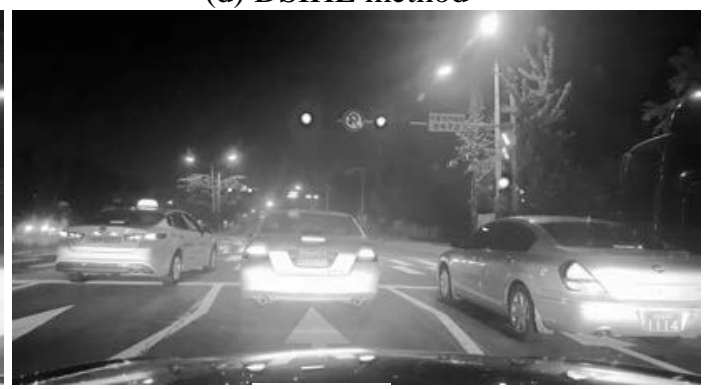

(f) Proposed algorithm

Fig. 8. Simulation results of conventional and proposed method for Night street image

Vehicle personal recorder images were employed to compare the proposed algorithm to existing algorithms. Table 1 shows the average and the standard deviation brightness of the proposed algorithm and the conventional algorithms such as Histogram Equalization (HE), Bi-Histogram Equalization (BBHE), Dualistic Sub-Image Histogram Equalization (DSIHE), and Dynamic Range Separate Histogram Equalization (DRSHE). It includes the total number 
(the product of brightness value and its pixel number), the average brightness, and the standard deviation brightness. The greater the Total number is, the more the number of bright pixels. And the larger standard deviation means more evenly distributed brightness relative to the average. Simulation results of $\mathrm{HE}$ and BBHE show lower values than the other algorithms, which means darker images. Although the proposed algorithm has Total Number value close to DRSHE and DSIHE, it has much larger standard deviation than those. That is, a histogram distribution of the proposed algorithm may be more widely distributed than the DRSHE and DSIHE.

Table 1. The average and the standard deviation brightness of the algorithms

\begin{tabular}{|c|c|c|c|c|c|c|}
\hline & \multicolumn{2}{|c|}{ Total Number(unit : Million) } & \multicolumn{2}{|c|}{ Average } & \multicolumn{2}{|c|}{ Standard Deviation } \\
\hline Algorithm & $\begin{array}{l}\text { Night } \\
\text { street }\end{array}$ & $\begin{array}{l}\text { Underground } \\
\text { parking lot }\end{array}$ & $\begin{array}{l}\text { Night } \\
\text { street }\end{array}$ & $\begin{array}{l}\text { Underground } \\
\text { parking lot }\end{array}$ & $\begin{array}{l}\text { Night } \\
\text { street }\end{array}$ & $\begin{array}{l}\text { Underground } \\
\text { parking lot }\end{array}$ \\
\hline Original & 61 & 77 & 66.52 & 83.33 & 0.55 & 0.54 \\
\hline $\mathrm{HE}$ & 92 & 927 & 99.41 & 100.02 & 0.77 & 0.68 \\
\hline DRSHE & 112 & 100 & 121.18 & 108.34 & 0.85 & 0.71 \\
\hline BBHE & 71 & 84 & 77.25 & 91.02 & 0.53 & 0.55 \\
\hline DSIHE & 109 & 105 & 118.37 & 113.48 & 0.67 & 0.65 \\
\hline \multirow[t]{2}{*}{ Proposed } & 114 & 113 & 123.16 & 122.93 & 1.67 & 1.73 \\
\hline & $\begin{array}{l}\text { Rainy night } \\
\text { street }\end{array}$ & $\begin{array}{c}\text { Out of the } \\
\text { tunnel }\end{array}$ & $\begin{array}{l}\text { Rainy night } \\
\text { street }\end{array}$ & $\begin{array}{l}\text { Out of the } \\
\text { tunnel }\end{array}$ & $\begin{array}{l}\text { Rainy night } \\
\text { street }\end{array}$ & $\begin{array}{l}\text { Out of the } \\
\text { tunnel }\end{array}$ \\
\hline Original & 29 & 459 & 31.25 & 49.07 & 0.55 & 0.44 \\
\hline $\mathrm{HE}$ & 120 & 113 & 129.86 & 123.15 & 0.86 & 1.84 \\
\hline DRSHE & 91 & 77 & 98.72 & 83.91 & 0.79 & 0.68 \\
\hline BBHE & 62 & 57 & 67.62 & 61.63 & 0.53 & 0.50 \\
\hline DSIHE & 117 & 96 & 127.38 & 103.97 & 0.67 & 0.59 \\
\hline Proposed & 83 & 101 & 90.60 & 109.71 & 1.13 & 2.92 \\
\hline
\end{tabular}

\subsection{License plate Capture}

Fig. 9 shows the flow chart of the license plate algorithm. First, it should remove the noise in the enhanced image within a median filter using a 3x3 mask. Second, it convert the gray image into the corresponding binary image to find connected components and then apply morphology algorithm. Then, we apply morphology operation to binary image. Dilation is an operation that grows or thickens objects in a binary images. 


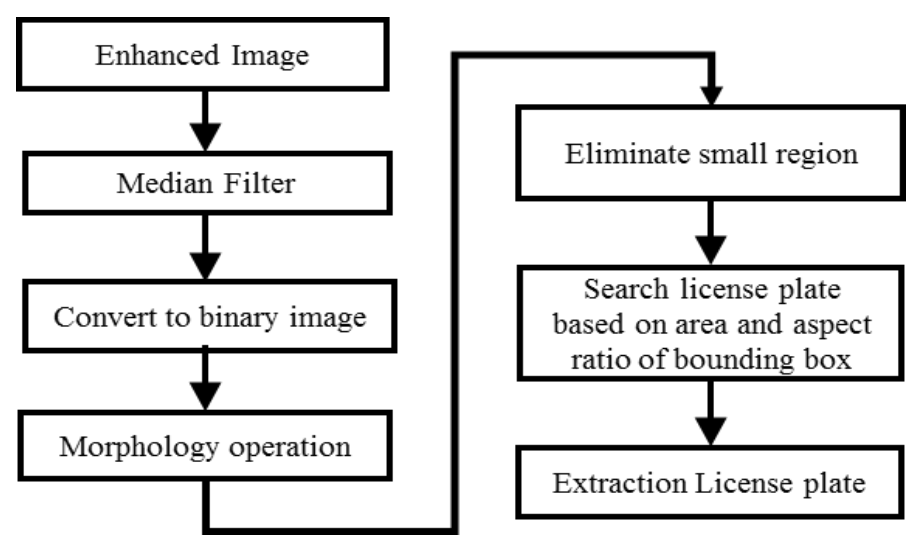

Fig. 9. Flowchart of the proposed algorithm

The dilation of A by B, denoted $A \oplus B$. The image was applied for an Eq. (23) as input image in the binary image.

$$
A \oplus B=\left\{Z \mid(\hat{B})_{Z} \cap A \neq \varnothing\right\}
$$

where $\varnothing$ is the empty set, $A$ is a selected block of the image, and $B$ is the structuring element[9]. After the experiment we use a linear structural element of length is 5 . By observing simulation results, we find that there are many small regions in the image, which are clearly not the license plate region. So we can use the bwareaopen function of MATLAB to eliminate these regions. The results show that these regions can be removed well by setting the area threshold to 5,000 or 10000 pixels, where the area threshold value may vary according to the image resolution. We can find that the majority of non-plate regions have been eliminated, but there are still holes in the remaining two regions, which require morphological operation to further process the image. In morphological image processing, the morphological image closing of $\mathrm{A}$ by $\mathrm{B}$, denoted $A \circ B=\{A \Theta B\} \oplus B$, is a dilation followed by an erosion: [9]

$$
A \circ B=\bigcup\left\{(B)_{Z} \mid(B)_{Z} \subseteq A\right\}
$$

Since the morphological closing tends to smooth the contours of object, it generally joins narrow breaks, fills long thin gulfs, and fills holes smaller than structuring element. After the experiment we use a 5 by 5 square structure element to do morphological closing on the image.
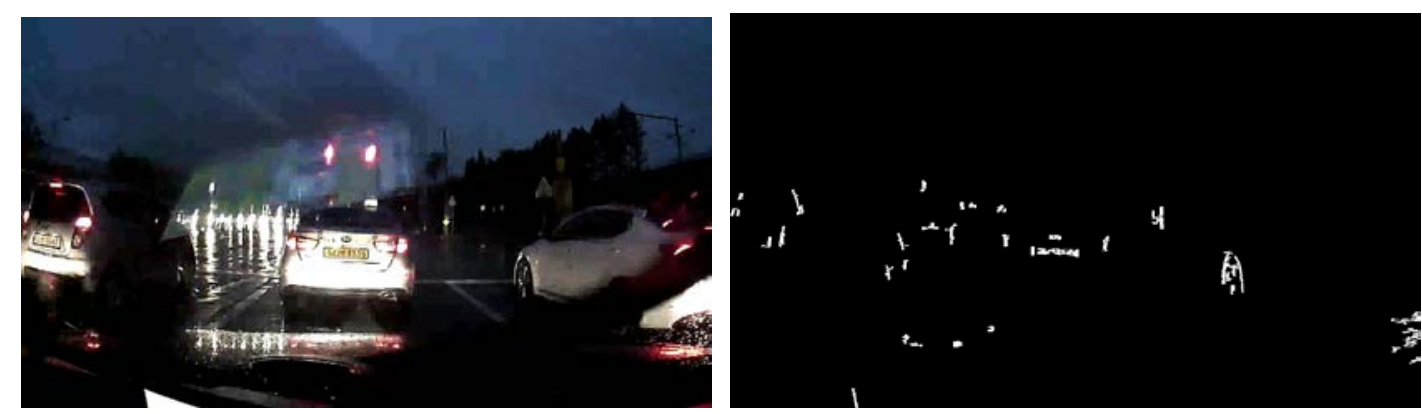

Fig. 10. Simulation results of threshold value for Rainy night street image 
Fig. 10 shows a simulation result of Eq. (24) in binary image. In Fig. 10, we can find the white shapes which are not a part of the license plate. And those should be deleted because these are not license plate shape. Then, the box filter is used to blur the edge image before calculating the energy map of the edges. The kernel $K$ in Eq. (25) is used. For applying threshold to find the large energy region, we should calculate the threshold value.

$$
\begin{aligned}
K & =\gamma\left|\begin{array}{cccc}
1 & 1 & \ldots & 1 \\
1 & 1 & \ldots & 1 \\
\ldots & \ldots & \ldots & \ldots \\
1 & 1 & \ldots & 1
\end{array}\right| \\
\gamma & =\left\{\begin{array}{cc}
\frac{1}{\text { ksize } \bullet \text { width } * \text { ksize } \bullet \text { height }} & \text { nomalize }=\text { true } \\
1 & \text { otherwise }
\end{array}\right.
\end{aligned}
$$

where ksize is the smoothing kernel size and normalize indicates whether the kernel is normalized by its area or not [23].

\section{4. simulation result}

The simulation has been performed using a notebook computer (CPU : i5-5200U 2.2Ghz, RAM : 8.00GB), Visual Studio 2010, OpenCV 2.10, and Matlab2016b.

We have used more than 500 moving images for verification of the proposed algorithm. Test images were captured from the device mounted in the car while driving in various environments. The images have been recorded in one minute increments. Algorithm verification is not a real time operation, but computerized simulation is carried out by transferring recorded images using portable memory. In the further research, the experiments will be performed by directly connecting the video storage device and the computer.

Fig. 11 shows the simulation results of a license plate extraction of the proposed algorithm and conventional algorithms (HI in Fig. 11(b), BBHE in Fig. 11(c), DSIHE in Fig. 11(d)) and DRSHE in Fig. 11(e)). The conventional algorithms resulted in extracting too large region or wrong region. In other words, they have extracted the license plate with other unnecessary regions, since they extended the histogram distribution excessively. However, the proposed algorithm resulted in less excessive and further limited enhancement in the bright area than conventional algorithms. That is, it has maintained the original characteristics of the image, i.e., the proposed algorithm could extract the car license plate region only. 


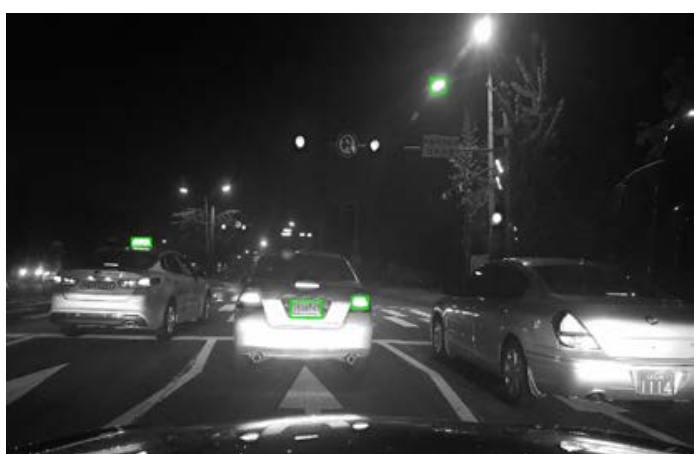

(a) Original Image

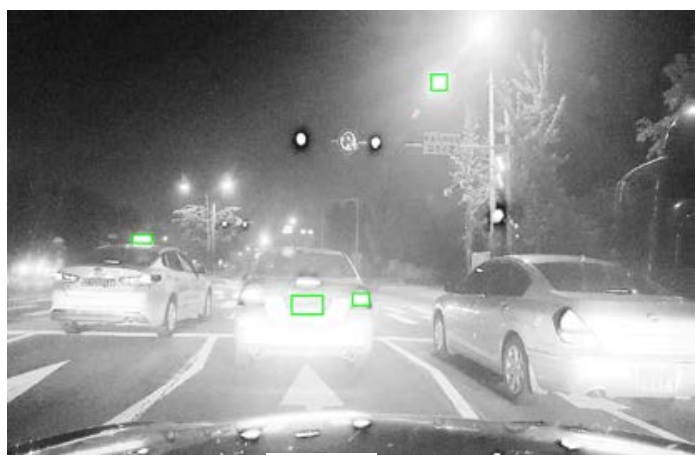

(c) BBHE method

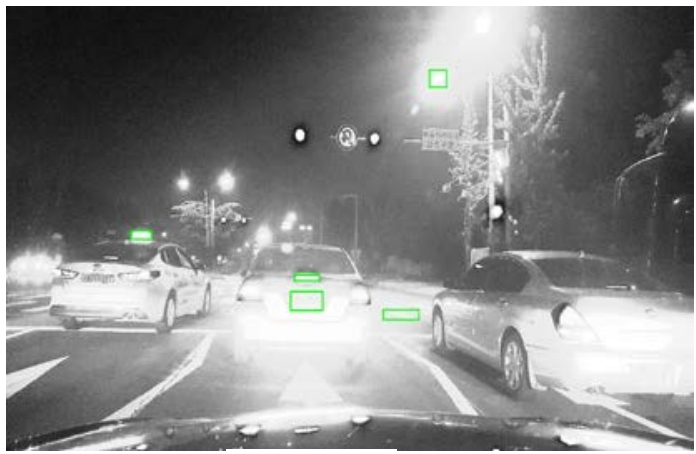

(e) DRSHE method

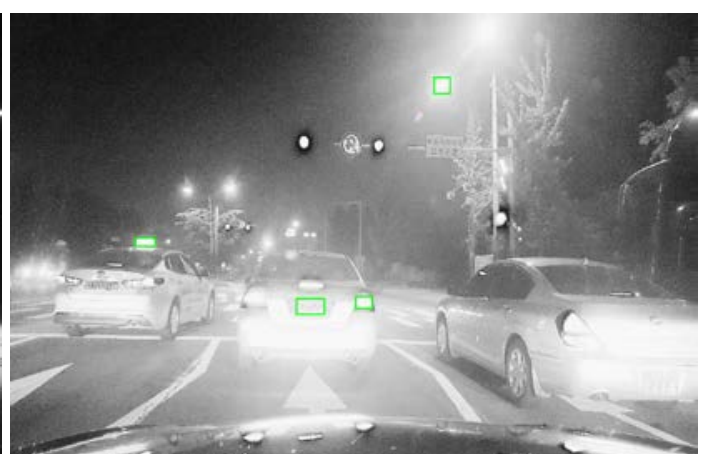

(b) Histogram Equalization method

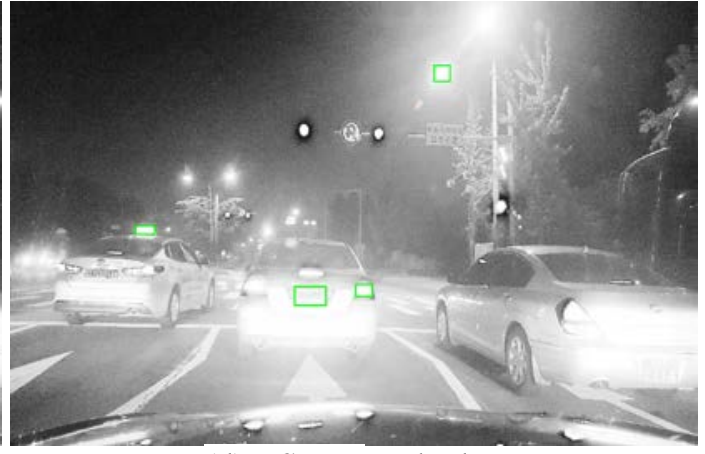

(d) DSIHE method

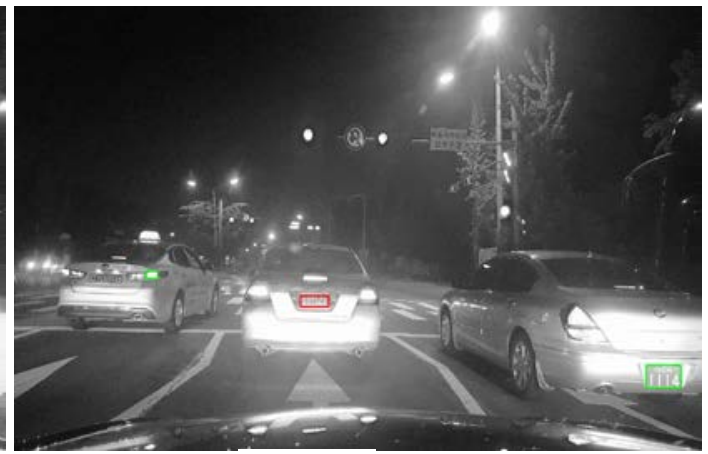

(f) Proposed algorithm

Fig. 11. Simulation results of Night street image

Fig. 12 shows the license plate extraction results. In Fig. 12(a), Day Street 1 image includes 2 license plates, and the proposed algorithm extracts all the license plates perfectly. In Fig. 12 (b), Day Street 2 image has 4 license plates. Simulation results show that all 4 extracted license plates and their license plate characters look clear. In Fig. 12 (c), 
Extracted license plates of original image extracted license plates by proposed algorithm

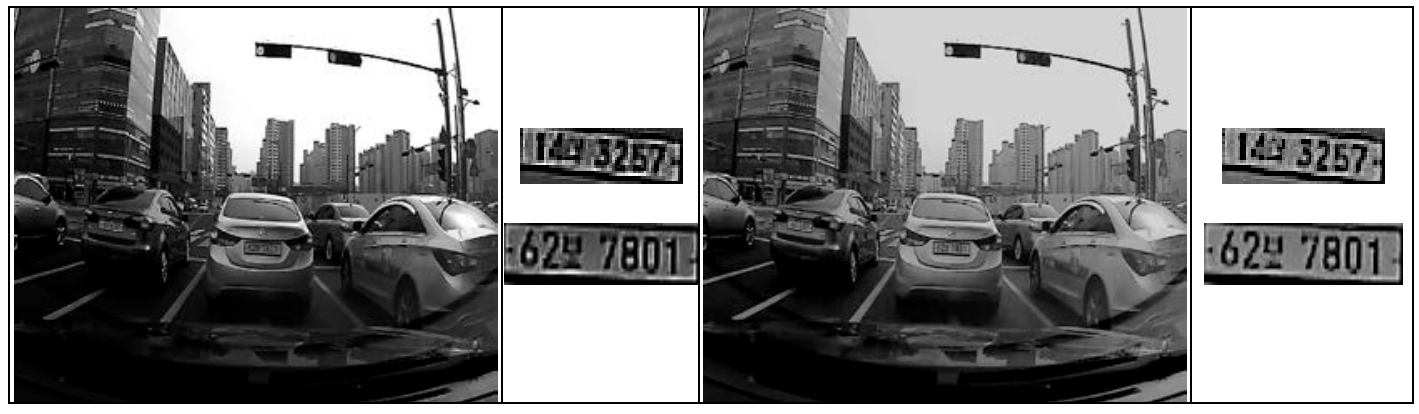

(a) Day street 1(2 license plates) and license plate extraction
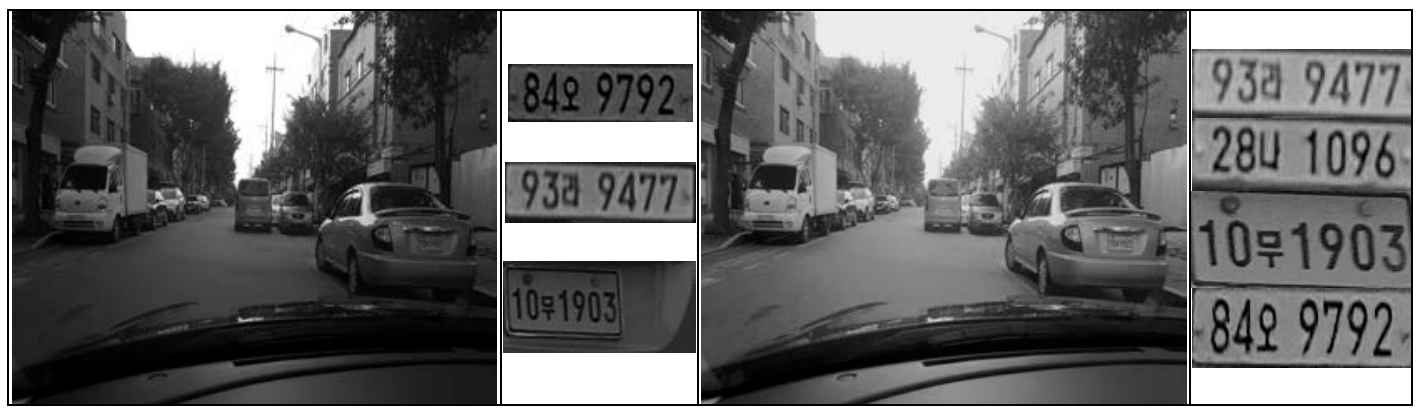

(b) Day street 2(4 license plates) and license plate extraction

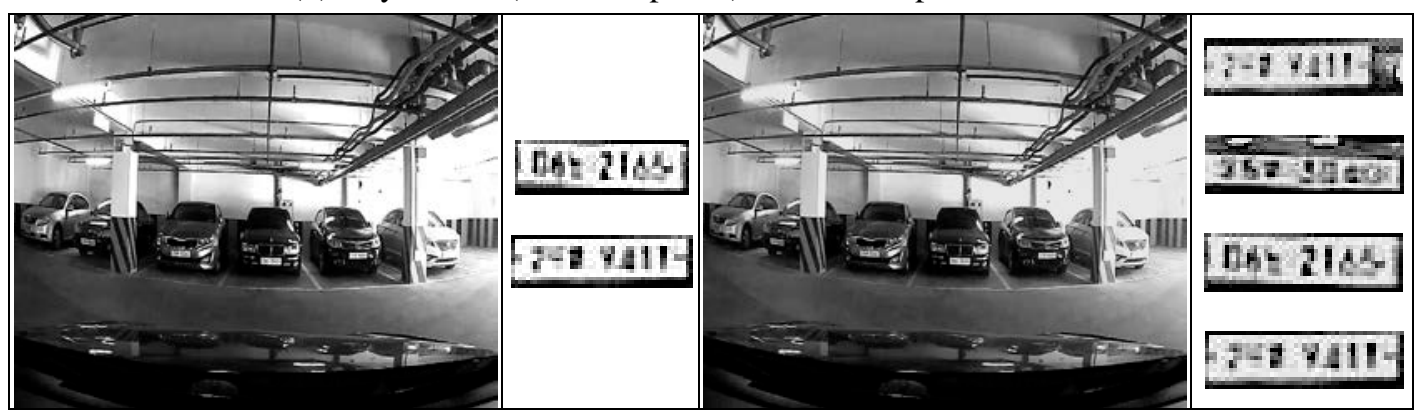

(c) Underground parking lot(5 license plates) and license plate extraction

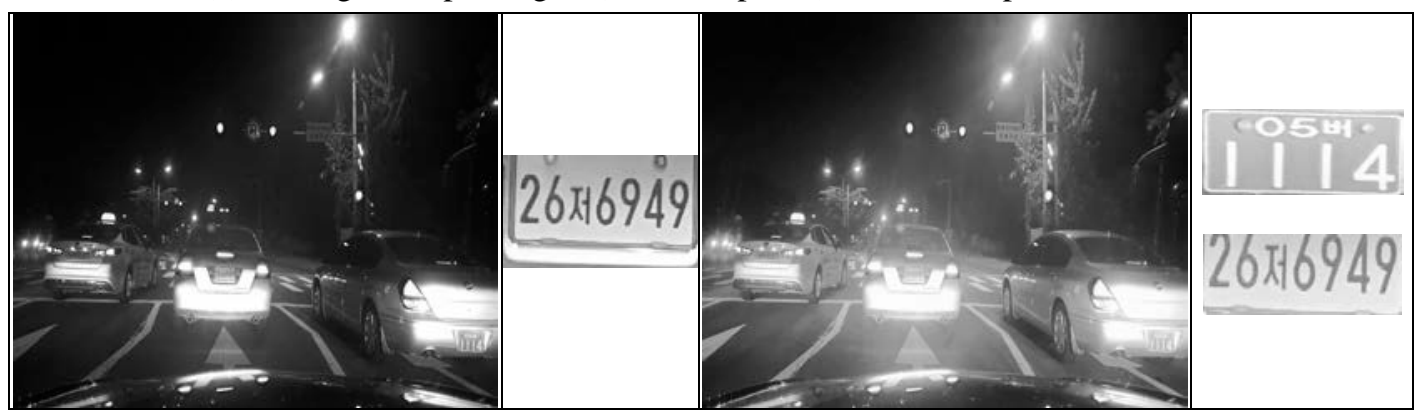

(d) Night street(3 license plates) and license plate extraction

Fig. 12. Simulation results of license plate extraction

Underground parking image has 5 identifiable license plates, but the simulation results show 4 extracted license plates. And the characters of the extracted license plate cannot be 
seen clearly due to long distance between the camera and the license plate, and the relatively low captured image resolution. In Fig. 12 (d), Night street image has 3 license plates. And only 2 license plates were extracted due to the long distance between the camera and the license plate. In order to achieve better results, further research should consider the factors such as the distance between the camera and license plate and the resolution of the captured images.

Table 2 summarizes the license plate extraction success rate of 3 different environmental images with 1 to 4 extracted license plates, which are chosen by visual inception. That is, simulation was performed in three environments (Day Street, Night Street, and Underground Parking Lot). And more than 100 images were used for each environment. As a result, the night street images among three environments resulted in the lowest extraction success rate $78.9 \%$. The reason was due to external environments such as street lights, darkness and vehicle headlights. Due to reflected headlights, the license plates could not be extracted even at close distances. Also, the lighting of underground parking lot affects license plate extraction success rate of the underground parking lot images. HE and DRSHE were the same as the extraction success rate of original images. BBHE and DSIHE were improved to $5.5 \%$ and $3 \%$ higher than the original extraction rate, respectively. However, the proposed algorithm resulted in license plate extraction success rate, $88.4 \%$. We found that license plate extraction success rate is influenced by the external environment such as the distance between vehicle and vehicle headlight reflection, license plate type, street light and the vehicle recording device such as camera lens and image resolution.

Table 2. License plate extraction success rate (unit : \%)

\begin{tabular}{|c|c|c|c|c|c|c|}
\hline Environment & Original & $\begin{array}{c}\mathrm{HE} \\
{[7]-[10]}\end{array}$ & $\begin{array}{c}\text { DRSHE } \\
{[10]}\end{array}$ & $\begin{array}{c}\text { BBHE } \\
{[12]}\end{array}$ & $\begin{array}{c}\text { DSIHE } \\
{[11]}\end{array}$ & Proposed \\
\hline \hline Day street & 69.2 & 69.2 & 69.2 & 76.9 & 69.2 & 92.3 \\
\hline Night street & 57.6 & 57.6 & 57.6 & 60.6 & 60.6 & 78.9 \\
\hline $\begin{array}{c}\text { Underground } \\
\text { parking lot }\end{array}$ & 64.7 & 64.7 & 64.7 & 70.6 & 70.6 & 94.1 \\
\hline $\begin{array}{c}\text { Extraction } \\
\text { success rate }\end{array}$ & 63.8 & 63.8 & 63.8 & 69.3 & 66.8 & 88.4 \\
\hline
\end{tabular}

\section{Conclusion}

We proposed an image enhancement method for extracting multi-license plate region. With the proposed image enhancement algorithm, we can get more detailed images by using the probability distribution value which reduces the excessive brightness. The enhancement factor was used to emphasize the license plate region of the captured image while preserving the original image characteristics. We have used more than 500 moving images for verification of the proposed algorithm.

The simulation results showed that the license plate extraction success rate is $20 \%$ higher than conventional image enhancement methods. However, the license plate extraction success rate of night street images was a little lower than that of other environments due to the reflected backlight. The distance between the camera and license plate and the resolution of the captured images might affect the exact appearance of the recognized characters of the 
extracted license plate. Future work might include the study of factors affecting the character recognition such as the distance between the camera and license plate and the resolution of the captured images. The proposed algorithm might be suitable for real-time implementation for ITS applications.

\section{References}

[1] Christos-Nikolaos E Anagnostopoulos, Ioannis E. Anagnostopoulos, Ioannis D. Psoroulas, Vassili Loumos, and Eleftherios Kayafas, "License Plate Recognition From Still Images and Video Sequences: A Survey," IEEE Trans. On Intelligent Transportation Systems, VOL. 9, pp 377 391, 2008. Article(CroRef Link)

[2] Yingjun Wu, Shouxun Liu and Xuan Wang.,"License Plate Location Method Based on Texture and Color," in Proc. of IEEE International Conference on Software Engineering and Service Science (ICSESS), PP.361-364, 2013. Article(CroRef Link)

[3] Hao Sheng, Chao Li, Qi Wen and Zhang Xiong. "Real-Time Anti-InterferenceLocation of VehicleLicense Plates Using High-Definition Video," IEEE Intelligent Transportation Systems Magazine, pp.17-23, 2009. Article(CroRef Link)

[4] Suwa, M., Wu, Y., Kobayashi, M., Kimachi, M, Ogata, S., "A stereobased vehicle detection method under windy conditions," in Proc. of Intelligent Vehicles Symposium, IV 2000. Proceedings of the IEEE, pp.246-248, 2000. Article(CroRef Link)

[5] Robert, K. "Video-based traffic monitoring at day and night vehicle features detection tracking," in Proc. of 12th International IEEE Conference on Intelligent Transportation Systems, pp.I-6, 2009. Article(CrossRef Link)

[6] J. A. G. Nijhuis, M. H. T. Brugge, K. A. Helmholt, J. P. W. Pluim, L. Spaanenburg,R. S. Venema, and M. A. Westenberg, "Car license plate recognitionwith neural networks and fuzzy logic," in Proc. of IEEE Int. Conf Neural Networks, vol. 5, pp. 2232-2236, 1995.

Article(CroRef Link)

[7] R. Crane, Simplified Approach to Image Processing, Prentice-Hall, pp. 55-83, 1994. Article(CroRef Link)

[8] R. C. Gonzalez, Digital Image Processing, Prentice-Hall, pp. 79-108, 2002. Article(CroRef Link)

[9] R. C. Gonzalez, Digital Image Processing, Prentice-Hall(Third Edition), pp. 649 - 702, 2010. Article(CroRef Link)

[10] Bernd jähne, Digital Video Processing, Springer-Verlag, pp. 77-94, 1993. Article(CroRef Link)

[11]Y. Koo, et al., "An Image Resolution Enhancing Technique Using Adaptive Sub-Pixel Interpolation for Digital Still Camera system," IEEE Trans. On Consumer Electronics, Vol. 45, No. 1, pp. 118-122, 1999. Article(CrossRef Link)

[12]Y. T. Kim, et al., "Contrast Enhancement Using Brightness Preserving Bi-Histogram Equalization," IEEE Trans. On Consumer Electronics, Vol. 43, No.1, pp. 1-8, Feb. 1997. Article(CrossRef Link)

[13] S. Y. Kim, et al., "Image Contrast Enhancement Based on the Piecewise-Linear Interpolation of CDF," IEEE Trans. On Consumer Electronics, Vol. 45, No. 3, pp. 828-834, Aug. 1999. Article(CrossRef Link)

[14]G.-H. Park et al., "A Contrast Enhancement Method using Dynamic Range Separate Histogram Equalization," IEEE Transactions on Consumer Electronics, Vol. 54, No. 4, pp.1981-1987, NOVEMBER 2008. Article(CroRef Link)

[15]K. N. Platanioits, Color Image Processing and Application, Springer, pp. 209-229, 2000. Article(CrossRef Link)

[16] H. C. Kim, et al., "An image interpolator with image improvement for LCD controller," IEEE Trans. On Consumer Electronics, Vol. 47, pp. 263-271, May 2001. Article(CrossRef Link) 
[17]F. G. Stremler, Introduction to Communication Systems, Addison-Wesley, pp. 459-486, 1993. Article(CroRef Link)

[18] K. N. Plataniotis, Color Image Processing and Application, Springer, pp. 32-40, 2000. Article(CrossRef Link)

[19]R. Bala, et al., "Gamut Mapping to Preserve Spatial Luminance Variations," Journal of Image Science and Technology, Vol. 45, No. 5, pp. 436-443, September/October 2001.

Article(CroRef Link)

[20]C. S. Lee, et al., "Gamut Mapping Algorithm Using Lightness Mapping and Multiple Anchor Points for Linear Tone and Maximum Chroma Reproduction," Journal of Image Science and Technology, Vol. 45, No. 3, pp. 209-223, May/June 2001. Article(CroRef Link)

[21] H. S. Chen and H. Kotera, "Three- dimensional Gamut Mapping Method Based on the Concept of Image Dependence," Journal of Image Science and Technology, Vol. 46, No. 1, pp. 44-52, January/ February 2002. Article(CroRef Link)

[22] Haoliang Li, Tao Qin. "A License Plate Location Algorithm based on Multicomponent Edge Combination of the HSI color space," in Proc. of IEEE International Congress on Image and Signal Processing, vol. 2, pp.1127-1129, 2011. Article(CroRef Link)

[23] Mostafa Kamal Sarker, et al., "Novel License Plate Detection Method Based on Heuristic Energy Map," J-KICS, vol 38C No.12, pp. 1114-1125, 2013. Article(CroRef Link)

[24] T. K. Kim et al., "Contrast enhancement system using spatially adaptive histogram equalization temporal filtering,” IEEE Trans. on Consumer Electronics, Vol.44, No 1, pp. 82-87, 1998. Article(CrossRef Link)

[25] Soong-Der Chen, Abd. Rahman Ramli, "Minimum Mean Brightness Error Bi-Histogram Equalization in Contrast Enhancement," IEEE Trans. on Consumer Electronics, Vol.49, No. 4, pp. 1301-1319, Nov. 2003. Article(CrossRef Link).

[26] Young-tack Kim and Yong-hun Cho, "Image Enhancing Method Using Men-Separate Histogram Equalization,” United States Patent, Patent No. 5,963,665, Oct. 5, 1999. Article(CroRef Link)

[27] Y. Q. Li, "Application of adaptive histogram equalization to X-ray chest image," in Proc. of the SPIE, 2321: pp 513-514, 1944. Article(CrossRef Link)

[28] Yeong-taeg Kim, "Method For Image Enhancing Using Quantized Men-Separate Histogram Equalization,” United States Patent, Patent No. 5,857,033, Jan 5, 1999. Article(CroRef Link) 


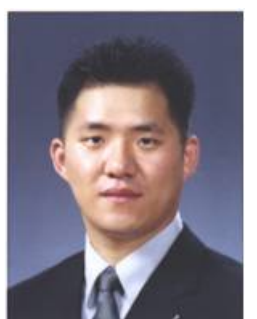

Jong-Ho Yun received the B.S. degree in electronic computer electrical and control from Hanyang University, Korea in 2001, and the M.S degree electrical control and instrumention from Hanyang University, Korea in 2003. He is currently a Ph.D. degree in electronic and computer engineering at Hanyang University, Korea. His areas of interests include image processing, image enhancement, Pattern recognition, license plate recognition and extraction and ASIC design..

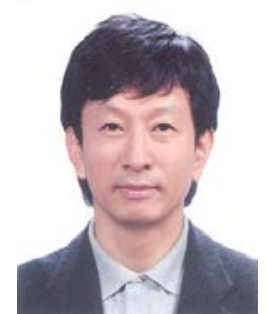

Myung-Ryul Choi (corresponding author) received the B.S. degree in electronic engineering from Hanyang University in 1983 and the M.S. and Ph. D. degrees in electrical engineering from Michigan State University in 1985 and 1991, respectively. From 1991 to 1992, he was with the Korea Institute of Industrial Technology where he was an assistant professor. In 1992, he joined the Department of Electrical Engineering and Computer Science at Hanyang University, where he is now a professor. His current research interests include digital/analog VLSI, 2D/3D FPD controller, RFID, ITS, and smart card applications.

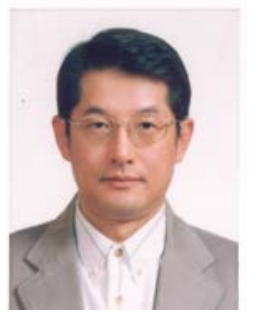

Sang-Sun LEE (corresponding author) received the B.S. degree in electronics engineering in 1978 and the M.S degree in electronic engineering in 1983, both from Hanyang University, Korea, and the Ph.D. degree in electronics engineering from University of Florida, USA in 1990. Now he has been a professor at the Department of Electronic Engineering, Hanyang University, Korea. His research interests include ubiquitous communication, standardizations, location based system, and vehicle-IT convergence techniques. 